\title{
Reconstruct the Heat Conduction Model with Memory Dependent Derivative
}

\author{
Wenwen Sun, Jinliang Wang \\ Research Institute for ESMD Method and Its Applications, College of Science, Qingdao University of Technology, Qingdao, China \\ Email: 895387872@qq.com, wangjinliang0811@126.com
}

How to cite this paper: Sun, W.W. and Wang, J.L. (2018) Reconstruct the Heat Conduction Model with Memory Dependent Derivative. Applied Mathematics, 9, 1072-1080.

https://doi.org/10.4236/am.2018.99072

Received: September 14, 2018

Accepted: September 25, 2018

Published: September 28, 2018

Copyright $\odot 2018$ by authors and Scientific Research Publishing Inc. This work is licensed under the Creative Commons Attribution International License (CC BY 4.0).

http://creativecommons.org/licenses/by/4.0/

(c) (i) Open Access

\begin{abstract}
The classical heat conduction equation is derived from the assumption that the temperature increases immediately after heat transfer, but the increase of temperature is a slow process, so the memory-dependent heat conduction model has been reconstructed. Numerical results show that the solution of the initial boundary value problem of the new model is similar to that of the classical heat conduction equation, but its propagation speed is slower than that of the latter. In addition, the propagation speed of the former is also affected by time delay and kernel function.
\end{abstract}

\section{Keywords}

Partial Differential Equation, Boundary Value Problem, Memory-Dependent Derivative (MDD), Caputo Type Fractional Derivative, Heat Conduction Equation

\section{Introduction}

Fractional calculus is an important branch of mathematics. The original fractional differential operator has three forms: Grumwald-Letnikov definition, Riemann-Liouville definition and Caputo definition. Caputo type fractional derivative is more convenient to solve practical problems. In 2011, Wang \& $\mathrm{Li}$ [1] referring to Caputo type fractional derivative proposed a new derivative called memory-dependent derivative. Compared with fractional derivative, its kernel function can be selected according to actual conditions and the memory-dependent interval does not increase with time $t$. It always concentrates the dependency interval on a limited time period related to the past state $[t-\tau, t]$, here $\tau$ is a time delay. The concept of derivative has attracted many scholars' attention. Ezzat et al. used memory-dependent derivatives in generalized thermos-viscoelasticity in [2] [3] [4]. In addition, Wang \& Li put forward memo- 
ry-dependent differential equations and studied them deeply in [5]. In [6], we studied the classical string vibration equation and heat conduction equation. The time derivative is replaced by the memory-dependent derivative. The numerical results simulated by MATLAB are between the wave equation and the heat conduction equation. With time, both the wave propagation and the energy attenuation occur. This change conforms to the characteristic of the "weighted period" function [7]. The diffusion velocity is lower than the heat conduction equation, and the wave amplitude is much higher than the wave equation under the same initial condition and boundary condition. Compared with the numerical solutions of fractional partial differential equations, the attenuation speed of the numerical solutions is slower and the wave phenomenon is more obvious.

The above research is only a simple substitution for the time derivative, and does not construct a model from the actual physical background. The classical heat conduction equation has obvious physical background. Its setting is derived without considering the motion of the medium, when the temperature rises immediately in a certain region of heat transfer, the problem of infinite velocity will arise.

From the definition of memory-dependent derivative, we can see that it is reflected in the average of the overall rate of change over a period of time, compared with the ordinary derivative, it can reflect the dependence of physical process on past state. It is more in line with the physical fact that the temperature rises slowly when heat propagates in the medium of less dense gas in theory. Is the heat conduction model made of it more realistic? In this paper, we use modeling method to explore.

\section{New Heat Conduction Model}

\subsection{Memory-Dependent Derivatives (MDD)}

Based on Caputo fractional differential operator

$$
D_{a}^{\alpha} f(t)=\int_{a}^{t} K_{\alpha}(t-s) f^{(m)}(s) \mathrm{d} s,
$$

here kernel function $K_{\alpha}(t-s)=(t-s)^{m-\alpha-1} / \Gamma(m-\alpha)$ and $f^{(m)}(t)$ represents the usual $m$-order derivative, Wang \& Li (2011) gives the definition of the memory-dependent derivative:

Definition 1 [1]: Let $m$ be a natural number $(m \in \mathrm{N})$, then for an $m$-times differentiable function $f(t)$

$$
D_{\tau}^{m} f(t)=\frac{1}{\tau} \int_{t-\tau}^{t} K(t-s) f^{(m)}(s) \mathrm{d} s,
$$

it is called m-order "memory-dependent derivative" of $f$ at $\tau$ relative to the time delay $\tau>0$, where $K(t-s)$ is a $m$-times differentiable about $t$ and $s$.

Compared with the derivative in Equation (2.1), the kernel function of the derivative in Equation (2.2) can be selected according to the actual situation. It is found by calculation in [1], Caputo-type derivatives will lead to the failure of long-time variation process under large time $t$, which is the inherent defect of 
this kind of derivatives, so memory-dependent derivatives are more reasonable.

We find that the memory-dependent derivative in Equation (2.2) is approximately equal to half of $f^{(m)}(t)$. In order to keep the values of memory-dependent derivative and ordinary derivative basically unchanged, an improved definition is given.

Definition 2 [6]: Let $m$ be a natural number $(m \in \mathrm{N})$, then for an $m$-times differentiable function $f(t)$

$$
D_{\tau}^{m} f(t)=\frac{1}{\int_{t-\tau}^{t} K(t-s) \mathrm{d} s} \int_{t-\tau}^{t} K(t-s) f^{(m)}(s) \mathrm{d} s,
$$

it is called $m$-order "memory-dependent derivative" of $f$ at $\tau$ relative to the time delay $\tau>0$, where $K(t-s)$ is a $m$-times differentiable about $t$ and $s$.

\subsection{Model Construction}

Considering that the temperature rises slowly with time after heat is transferred into an object, that is, the rise of temperature is delayed relative to the heat transfer process. From the physical process, a one-dimensional heat conduction model with memory-dependent derivatives on a cylinder is constructed. The specific derivation process is as follows.

Consider a cylinder (assuming that the cross section area is 1, the object is homogeneous and isotropic, without considering thermal expansion). A function $u(x, t)$ is used to represent the temperature at the position $x$ and $t$ at the cylinder. As shown in Figure 1, an infinitesimal segment $[x, x+\Delta x]$ is selected on the cylinder, think heat enters from $x=0$ segment. According to the Fourier law of heat transfer, The heat $\mathrm{d} Q$ of a body flowing through an infinitesimal small area $\mathrm{d} S$ (assuming $\mathrm{d} S=1$ is the cross-sectional area of a cylinder) in the direction $n$ along the normal in an infinitesimal time increment $\mathrm{d} t$ (discretized equivalent to $\Delta t$ ) is proportional to the directional derivative $\partial u / \partial n$ of the body temperature along the normal direction of the surface $\mathrm{d} S$, That is

$$
\mathrm{d} Q=-\kappa(x) \frac{\partial u}{\partial n} \mathrm{~d} S \mathrm{~d} t,
$$

here $k(x)$ is called the thermal conductivity of the object at point $x$, positive value should be taken. The negative sign in Equation (2.4) indicates that heat always flows from the side of high temperature to the side of low temperature. Therefore, $\mathrm{d} Q$ should have a different sign from $\partial u / \partial n$. Discrete the Equation (2.4) and consider the amount of heat flowing into $[x, x+\Delta x]$ during the period of $\tau(\tau=n \Delta t)$, the amount of heat change is

$$
\Delta Q=-\kappa \frac{u(x+\Delta x, t)-u(x, t)}{-\Delta x} \Delta S \tau
$$

The influx of heat changes the temperature inside the body, but the increase in temperature is a slow process. Suppose it will delay $\tau$ periods. When temperature is changed from $u(x, t)$ to $u(x, t+\tau)$, the amount of heat change is 


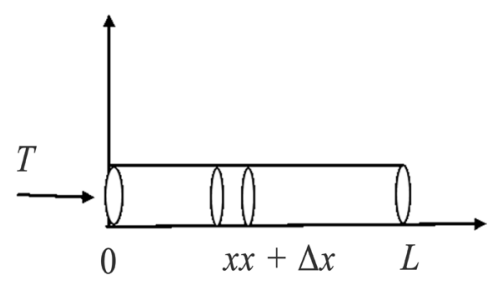

Figure 1. Schematic diagram of thermal incoming process.

$$
\begin{aligned}
\Delta Q= & c \rho \Delta S \Delta x\left\{k_{1}[u(x, t+\Delta t)-u(x, t)]+k_{2}[u(x, t+2 \Delta t)-u(x, t+\Delta t)]\right. \\
& \left.+\cdots+k_{n}[u(x, t+\tau)-u(x, t+(n-1) \Delta t)]\right\},
\end{aligned}
$$

here $c$ is the specific heat, $\rho$ is density, $k_{m}(m=1,2, \cdots, n)$ indicates the effect of temperature on the final temperature in small time interval $\Delta t$. The $[t, t+\Delta t]$ time period effect should be minimal and the $[t+(n-1) \Delta t, t+\tau]$ time period has the greatest impact, this is related to time $t$ and delay $\tau$. Available from Equation (2.5) and Equation (2.6), have to

$$
\kappa \frac{u(x+\Delta x, t)-u(x, t)}{\Delta x} \tau=c \rho \Delta x \sum_{m=1}^{n} k_{m} \frac{u(x, t+m \Delta t)-u(x, t+(m-1) \Delta t)}{\Delta t} \Delta t .
$$

Collate

$$
\frac{\kappa}{c \rho} \frac{u(x+\Delta x, t)-u(x, t)}{(\Delta x)^{2}}=\frac{1}{\tau} \sum_{m=1}^{n} k_{m} \frac{u(x, t+m \Delta t)-u(x, t+(m-1) \Delta t)}{\Delta t} \Delta t .
$$

Order $\Delta x \rightarrow 0, \Delta t \rightarrow 0$ and $a^{2}=\kappa /(c \rho)$, have to

$$
a^{2} \frac{\partial^{2} u}{\partial x^{2}}=\frac{1}{\tau} \int_{t+\tau}^{t} k(t-s) \frac{\partial u}{\partial s} \mathrm{~d} s
$$

here, $k(t-s)$ is equivalent to the continuous form of $k_{m}(m=1,2, \cdots, n)$, which is related to time $t$ and delay $\tau$.

In particular, when $\tau \rightarrow 0$, there should be

$$
\left.\frac{1}{\tau} \int_{t}^{t+\tau} k(t-s) \frac{\partial u}{\partial s} \mathrm{~d} s \rightarrow \frac{\partial u}{\partial s}\right|_{s=t} .
$$

when $\tau$ is small, we apply the mean value theorem for the

$$
\begin{gathered}
\int_{t}^{t+\tau} k(t-s) \frac{\partial u}{\partial s} \mathrm{~d} s / \tau, \quad \exists \xi \in[t, t+\tau], \\
\frac{1}{\tau} \int_{t}^{t+\tau} k(t-s) \frac{\partial u}{\partial s} \mathrm{~d} s=\left.\frac{1}{\tau} \int_{t}^{t+\tau} k(t-s) \mathrm{d} s \cdot \frac{\partial u}{\partial s}\right|_{s=\xi} .
\end{gathered}
$$

Therefore, when $\tau \rightarrow 0, \int_{t}^{t+\tau} k(t-s) \mathrm{d} s / \tau \rightarrow 1$, In order to satisfy the minimum influence of time $t$ on the temperature, the time $t+\tau$ has the greatest influence, whether $\tau$ is small or not, it should be satisfied

$$
\frac{1}{\tau} \int_{t}^{t+\tau} k(t-s) \mathrm{d} s=1
$$

Mark $k(t-s)=C K(t-s)$, be 


$$
\frac{1}{\tau} \int_{t}^{t+\tau} C K(t-s) \mathrm{d} s=1
$$

have to

$$
C=\frac{\tau}{\int_{t}^{t+\tau} K(t-s) \mathrm{d} s} .
$$

Here, $K(t-s)$ is a core function that can be chosen according to needs, and bring $C$ back to (8)

$$
a^{2} u_{x x}=D_{\tau} u,
$$

among

$$
D_{\tau} u=\frac{1}{\int_{t}^{t+\tau} K(t-s) \mathrm{d} s} \int_{t}^{t+\tau} K(t-s) \frac{\partial u}{\partial s} \mathrm{~d} s
$$

equivalent to a forward first-order memory-dependent derivative and Equation (2.9) is a one-dimensional heat conduction model with first-order forward memory-dependent derivative.

\section{Numerical Simulation}

In the previous section, a memory-dependent heat conduction model is deduced. In this section, we mainly study the solution of the initial-boundary value problem under the first boundary condition, discuss the effect of time delay and kernel function on the solution and compare it with the classical heat conduction equation.

\subsection{The Initial Boundary Value Problem of the New Model}

The initial boundary value problem of the memory-dependent heat conduction model is discussed

$$
\begin{cases}a^{2} u_{x x}=D_{\tau} u \quad(0<x<L, 0<t<T) \\ u(x, 0)=\sin (\pi x) \quad(0<x<L) \\ u(0, t)=u(L, t)=0 \quad(0<t<T)\end{cases}
$$

among

$$
D_{\tau} u=\frac{1}{\int_{t}^{t+\tau} K(t-s) \mathrm{d} s} \int_{t}^{t+\tau} K(t-s) \frac{\partial u}{\partial s} \mathrm{~d} s,
$$

the kernel function $K(t-s)$ takes the form of a linear function, that is

$$
K(t-s)=\frac{s-t}{\tau} .
$$

Through calculation, $\int_{t}^{t+\tau} K(t-s) \mathrm{d} s=\tau / 2$. Easy to find, except diffusion coefficient $a$, time delay $\tau$ is the main factor affecting the numerical solution of problem (3.1).

The problem (3.1) is discretized by the finite difference method, take $\Delta t$ as 
time step, $\Delta x$ as space step, let $M=T / \Delta t, \quad N=L / \Delta x, \tau=n \Delta t$, $K_{i}=i / n, i=1,2, \cdots, n-1$ is the discrete form of the kernel function $K(t-s)=(s-t) / \tau, \quad r=a^{2} \cdot \tau /\left(2(\Delta x)^{2}\right)$, get down

$$
\left\{\begin{array}{l}
U_{j}^{i+n}=r U_{j+1}^{i}+\left(\frac{K_{1}}{K_{n}}-r\right) U_{j}^{i}-\frac{1}{K_{n}} \sum_{m=1}^{n-1}\left(K_{m}-K_{m+1}\right) U_{j}^{i+m}, \\
U_{j}^{0}=\sin (\pi j \Delta x), \quad j=1,2, \cdots, N-1, \\
U_{0}^{i}=U_{N}^{i}=0, \quad i=0,1,2, \cdots, M .
\end{array}\right.
$$

in the form of one $j=1,2, \cdots, N-1, i=0,1, \cdots, M-n$.

The temperature function of the first $n-1$ time period is used in numerical calculation, which is discrete form of classical heat conduction equation, That is

$$
U_{j}^{i+1}=\frac{a^{2} \Delta t}{(\Delta x)^{2}} U_{j+1}^{i}+\left(1-\frac{2 a^{2} \Delta t}{(\Delta x)^{2}}\right) U_{j}^{i}+\frac{a^{2} \Delta t}{(\Delta x)^{2}} U_{j-1}^{i}
$$

there $i=0,1, \cdots, n-2, j=1,2 \cdots, N-1$. When fixed $T=20, L=1$ and $a=0.1$, separately select $\tau=5 \Delta t, 150 \Delta t$ (When the $\Delta t$ is time step), the MATLAB software is implemented in Figure 2.

It is found from Figure 2 that the numerical solution function of the new model is also diffusive, and the effect on the global behavior of the solution is small when the delay $\tau$ is small (see (a) (b)). When the kernel function and the diffusion coefficient are fixed, the diffusion condition changes with time delay $\tau$. Because the temperature function in the first $n-1$ time period is given by the classical heat conduction equation, the temperature function in this $n-1$ time period can be regarded as the initial temperature. It can be found that the initial temperature decreases with the increase of time delay, which affects the diffusion. As can be seen from the image, the temperature decreases faster with the increase of time delay (see (c)). But considering the tangent slope at different times at the same location, it is found that the larger the time delay, the smaller the tangent slope. Reflects the increase in the rate of diffusion and slows down at any time. (see (d)).

\subsection{Comparison with Classical Heat Conduction Equation}

The heat conduction equation we know well.

$$
\begin{cases}u_{t}=a^{2} u_{x x} \quad(0<x<L, 0<t<T) \\ u(x, 0)=\sin (\pi x) & (0<x<L) \\ u(0, t)=u(L, t)=0 & (0<t<T)\end{cases}
$$

the numerical solution has obvious characteristics. Comparison questions (3.1) and problems (3.2).

The above problems are discretized by the finite difference method, and the following Figure 3 is achieved by MATLAB software.

As can be seen from Figure 3, the classical heat conduction equation is a 
(a) $\tau=5 \Delta t$

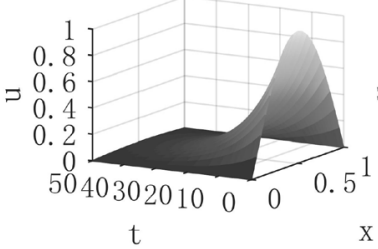

(c) $\mathrm{x}=0.5$

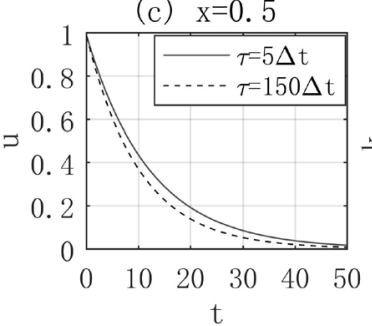

(b) $\tau=150 \Delta t$

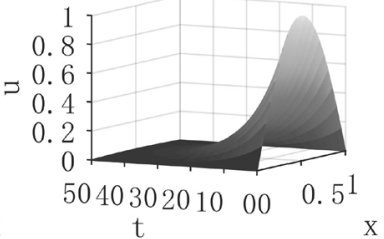

(d) Tangent slope at $x=0.5$

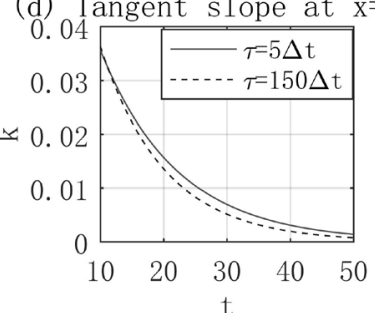

Figure 2. Influence of different delays on solutions.

(a)

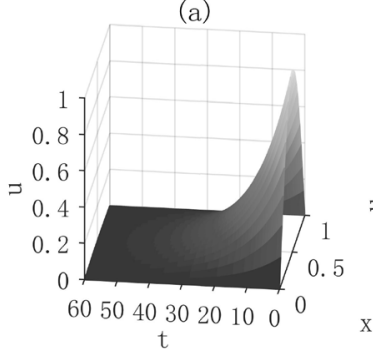

(b)

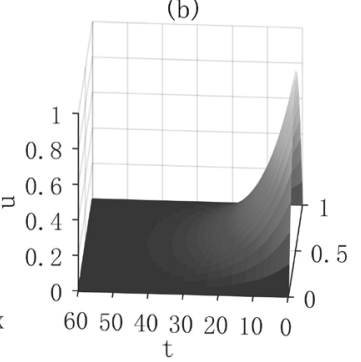

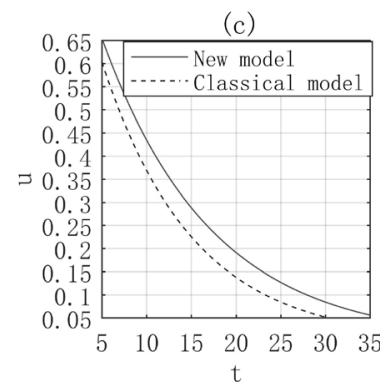

Figure 3. In case $a=0.1$, the numerical-simulating results for (a) new model with delay of 5 steps; (b) heat conduction equation; (c) particular case for this two kinds of problems with $x=0.5$.

diffusion equation with a fast diffusion rate over time (see (b)); the solution of the new model is similar to the classical heat conduction equation, and there is a diffusion phenomenon over time (see (a)), but the diffusion rate is slightly slower than that of the latter (see (c)). It is found that the new model is more effective in describing the real heat transfer phenomena.

\subsection{The Influence of Kernel Function on the New Model}

In the new model, the kernel function of memory-dependent derivative is a linear function and the effect of time delay on the solution is studied. By the definition of memory-dependent derivative, the kernel function is also one of the important factors affecting the solution. The influence of kernel function on solutions is discussed in this section. We mainly compare the influence of the kernel function on the nature of the solution when kernel function is a linear function and a quadratic function. The kernel function is chosen as follows.

$$
K_{1}(t-s)=\frac{s-t}{\tau}, K_{2}(t-s)=\left(\frac{s-t}{\tau}\right)^{2} .
$$

The discretization of the problems under different kernel functions is performed 
(a) $\mathrm{K}_{1}(\mathrm{t}-\mathrm{s})=(\mathrm{s}-\mathrm{t}) / \tau$

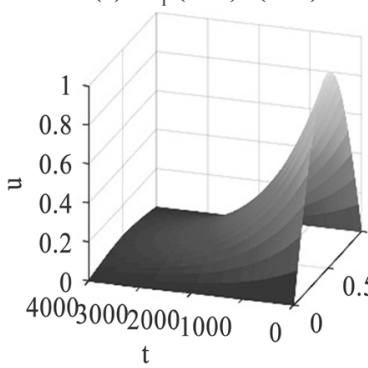

(b) $K_{2}(t-s)=((s-t) / \tau)^{2}$

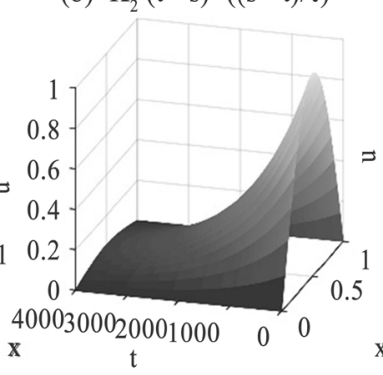

(c) $\mathrm{x}=0.5$

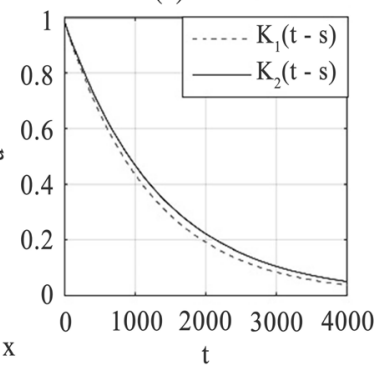

Figure 4. Influence of different kernel functions on solutions.

by the finite difference method, select $a=0.01, \tau=5 \Delta t$, using MATLAB to achieve Figure 4

As can be seen from Figure 4, the solutions under different kernel functions are roughly the same and the general behavior of the solutions remains the same, but the diffusion rates under different kernel functions are different (see (c)). When $a=0.01, \tau=5 \Delta t$ and the kernel function is a linear function, the diffusion rate is a little faster. The influence of different kernel functions on the solution still needs further study.

\section{Conclusion}

The classical heat conduction equation is proposed on the basis of the instantaneous rise of temperature after heat transfer. In fact, the rise of temperature is not instantaneous, but a slow process. Numerical results show that: 1) The solution of the new model is diffusion type. When the kernel function is unchanged, the diffusion rate slows down with the increase of time delay. 2) Compared with the classical heat conduction equation, the two properties are similar, but the diffusion rate of the new model is even slower. 3) It is found that the kernel function is also one of the factors affecting the diffusion rate, but how to influence the diffusion rate needs further study.

\section{Conflicts of Interest}

The authors declare no conflicts of interest regarding the publication of this paper.

\section{References}

[1] Wang, J.L. and Li, H.F. (2011) Surpassing the Fractional Derivative: Concept of the Memory-Dependent Derivative. Computers and Mathematics with Applications, 62, 1562-1567. https://doi.org/10.1016/j.camwa.2011.04.028

[2] Ezzat, M.A., El-Karamany, A.S. and El-Bary, A.A. (2016) Generalized Thermos-Viscoelasticity with Memory-Dependent Derivatives Involving Two Temperatures. Mechanics of Advanced Materials and Structures, 23, 545-553. https://doi.org/10.1080/15376494.2015.1007189

[3] Ezzat, M.A. and El-Bary, A.A. (2016) Thermoelectric MHD with Memory-Dependent Derivative Heat Transfer. International Communications in Heat 
and Mass Transfer, 75, 270-281.

https://doi.org/10.1016/j.icheatmasstransfer.2016.04.026

[4] Ezzat, M.A. and El-Bary, A.A. (2015) Memory-Dependent Derivatives Theory of Thermo-Viscoelasticity Involving Two-Temperature. Journal of Mechanical Science and Technology, 29, 4273-4279. https://doi.org/10.1007/s12206-015-0924-1

[5] Li, H.F. and Wang, J.L. (2012) Molding the Dynamic System with Memory-Dependent Derivative. 24th Chinese Control and Decision Conference (CCDC) Taiyuan, 23-25 May 2012, 23-25.

[6] Sun, W.W. and Wang, J.L. (2017) On Numerical Solution of the Memory Dependent Partial Differential Equations. Advances in Applied Mathematics, 6, 637-643. (in Chinese) https://doi.org/10.12677/AAM.2017.64074

[7] Wang, J.L. and Li, H.F. (2006) The Weighted Periodic Function and Its Properties. Dynamics of Continuous Discrete and Impulsive Systems (Series A-Mathematical Analysis), 13, 1179-1183. 